\title{
Sampled-Grating DBR Lasers: Calibrated 3D Simulation of Tuning Characteristics
}

\author{
Lutz Schneider ${ }^{a}$, Michael Pfeiffer ${ }^{c}$, Joachim Piprek ${ }^{b}$, Andreas Witzig ${ }^{c}$, and Bernd \\ Witzigmann $^{a}$ \\ ${ }^{a}$ Integrated Systems Laboratory, Swiss Federal Institute of Technology Zurich, ETHZ, \\ Gloriastrasse 35, CH-8092 Zürich, Switzerland; \\ ${ }^{b}$ ECE Department, University of California, Santa Barbara, CA 93106, USA; \\ ${ }^{c}$ Synopsys Switzerland Ltd., Affolternstrasse 52, CH-8050 Zürich, Switzerland
}

\begin{abstract}
We present a calibrated full-3D simulation of a widely-tunable sampled-grating distributed Bragg reflector (SGDBR) laser showing the characteristic quasi-continuous tuning map. The SGDBR laser is a longitudinally integrated device consisting of five waveguide sections: a front and rear mirror section together with a phase section allow for quasi-continuous tuning over a wavelength range of 100nm, while an active section provides the optical gain for the laser operation. For real world applications the tuning behavior needs to be well understood in order to guarantee stable operation for each wavelength channel. Due to the strong inhomogeneities both in the transverse and longitudinal dimensions a $3 \mathrm{D}$ simulation model is necessary to cover the full complexity of such devices. In our physics-based approach, we solve the fully coupled semiconductor drift-diffusion equations for electrons and holes, taking into account longitudinal current flux in full 3D. Gain calculation and the photon rate equation are included self-consistently in an iterative Newton scheme. The optical field is composed of several transverse mode patterns combined with the longitudinal field distribution as obtained by a transfer matrix formalism. By means of a Gummel-type iteration scheme a self-consistent solution of the optics and electronics is found. We show that this approach succeeds even in the numerically most challenging case of discrete wavelength jumps as observed in typical tuning maps of SGDBR lasers. Our simulations are in good agreement with measurements and prove the suitability of the simulator for the design and optimization of state-of-the-art tunable lasers.
\end{abstract}

Keywords: Tunable Lasers, SGDBR, Device Simulation, Laser Simulation, Quantum Well

\section{INTRODUCTION}

Widely-tunable semiconductor lasers are expected to play an important role in near-future optical communication applications and have already demonstrated their great potential when integrated with other optoelectronic devices on a single chip. ${ }^{1,2}$ It is a key technology in the development of future optoelectronic systems. A less spectacular application of such tunable lasers but economically more compelling is in the area of inventory reduction. Due to the recent telecoms downturn tunable laser manufacturers have also found sensing applications to be a fertile ground for their products.

The strong interaction between optical, electronic and thermal effects makes the design of photonic integrated devices and systems particularly challenging. Several devices are built on the same chip in close vicinity, making a separation into lumped models impossible. Modern technology computer aided design (TCAD) tools can be used for design and optimization of such complex devices. ${ }^{3}$ With the help of numerical models, the large parameter space can be scanned and development costs as well as time to market can be significantly reduced.

The immense increase in the computational power of modern workstations allows to solve the laser device equations of entire three-dimensional devices. A simulation scheme which fully reflects the 3D nature of tunable

Further author information: (Send correspondence to Lutz Schneider) Lutz Schneider: E-mail: lutz@iis.ee.ethz.ch, Telephone: +41 446327645 
multi-section DBR lasers is presented. It proves to be an efficient way of modeling ever more complex structures in 3D without compromising on the physics-based approach employed in 2D. The models chosen in this work aim to provide a balance between computational effort and the accuracy of the solution. The comprehensive simulation of multi-section lasers is becoming feasible, allowing to directly predict the optical device behavior together with the electronic performance. The simulation of unwanted effects such as longitudinal current leakage can be performed and therefore, the simulation tool is applicable for the optimization of several loss mechanisms.

In this simulator charge carrier transport is modeled by the drift-diffusion and Poisson equations. ${ }^{4}$ Temperature is included by an energy balance formalism, ${ }^{5}$ and the model for carrier transport at heterointerfaces and quantum wells is described in Refs. 6,7.

This paper is organized as follows. Section 2 briefly reviews the electro-thermal model employed and describes in detail the treatment of the three-dimensional optical problem, followed by a section on the numerical implementation of the physical models. Finally, in Sect. 4 simulation results are presented.

\section{PHYSICAL MODEL EQUATIONS}

\subsection{Electro-thermal model}

The electro-thermal simulation models are only summarized very briefly. Detailed descriptions can be found in Refs. 8 and 9 .

The basic equations of the thermodynamic model comprise the Poisson equation for the electrostatic potential $\phi$, and the continuity equations for the electrons $n$, holes $h$ and the local heat flux $\boldsymbol{S}$,

$$
\begin{aligned}
\nabla \cdot \boldsymbol{D} & =-q\left(p-n+N_{D}^{+}-N_{A}^{-}\right) \\
\nabla \cdot \boldsymbol{j}_{n} & =q\left(R+\partial_{t} n\right) \\
-\nabla \cdot \boldsymbol{j}_{p} & =q\left(R+\partial_{t} p\right) \\
\nabla \cdot \boldsymbol{S} & =-c_{t o t} \partial_{t} T-\nabla \cdot\left(\boldsymbol{j}_{n}\left(P_{n} T+\Phi_{n}\right)+\boldsymbol{j}_{p}\left(P_{p} T+\Phi_{p}\right)\right)
\end{aligned}
$$

The electron and hole quasi-Fermi potentials $\Phi_{n}$ and $\Phi_{p}$ can be directly calculated from the electron and hole densities. ${ }^{8}$ The variables $N_{D}^{+}$and $N_{A}^{-}$are the ionized donor and acceptor concentrations, and $P_{n}$ and $P_{p}$ denote the absolute thermo-electric powers for electrons and holes, respectively. The recombination rate $R$ is the sum of radiative and non-radiative recombination. Further equations define the electric displacement $\boldsymbol{D}$, the currents $j_{n}, j_{p}$, and the heat flux $S$

$$
\begin{aligned}
\boldsymbol{D} & =\varepsilon \nabla \phi \\
\boldsymbol{j}_{n} & =-q\left(\mu_{n} n \nabla \phi-D_{n} \nabla n+\mu_{n} n P_{n} \nabla T\right) \\
\boldsymbol{j}_{p} & =-q\left(\mu_{p} p \nabla \phi+D_{p} \nabla p+\mu_{p} p P_{p} \nabla T\right) \\
\boldsymbol{S} & =-\kappa_{t h} \nabla T,
\end{aligned}
$$

where $T$ is the local temperature, $\mu_{n}$ and $\mu_{p}$ are the mobilities and $D_{n}, D_{p}$ are the diffusion constants.

The Newton method using the well-known box discretization is applied for the numerical solution of the coupled nonlinear partial differential equations (PDEs). ${ }^{10}$ As shown above, (1)-(4), the semiconductor equations can be written in divergence-form equaling the divergence plus a scalar source term.

\subsection{Optical Model}

The optical model is based on a rate equation approach. For each optical mode, a separate rate equation describes the temporal evolution of the mean electro-magnetic energy. This approach is known as the adiabatic approximation $^{11}$ and is based on the assumption that the optical mode shapes depend on the instantaneous value of the time-dependent dielectric function. 
Starting from the time-domain Maxwell equations and employing the mode expansion

$$
\boldsymbol{E}(\mathbf{r}, t)=E_{0} \sum_{\nu} \Psi_{\nu}(\boldsymbol{r} ; t) \sqrt{S_{\nu}(t)} e^{-i \omega_{\nu} t} e^{-i \phi_{\nu}(t)}+\text { c.c. }
$$

in order to eliminate the fast time-scale, the photon rate equation can be written as ${ }^{8,11,12}$

$$
\partial_{t} S_{\nu}=\left(G_{\nu}-L_{\nu}\right) S_{\nu}+\beta_{\nu} R_{\nu}^{s p}
$$

where $G_{\nu}, L_{\nu}, \beta_{\nu}$ and $R_{\nu}^{s p}$ are the modal gain, effective modal loss, spontaneous emission coupling coefficient and spontaneous emission for mode number $\nu$, respectively. The constant $E_{0}$ in Eqn. (9) is chosen such that the energy stored in the optical field inside of the cavity is $W_{o p t}=\hbar \omega S$. The definition of the modal quantities is as follows

$$
\begin{aligned}
G_{\nu}(\omega) & =\int g^{l o c}(\mathbf{r}, \omega)\left|\Psi_{\nu}(\mathbf{r})\right|^{2} d \mathbf{r} \\
R_{\nu}^{s p}(\omega) & =\int r^{s p}(\mathbf{r}, \omega)\left|\Psi_{\nu}(\mathbf{r})\right|^{2} d \mathbf{r} \\
L_{\nu}(\omega) & =\int \alpha(\mathbf{r}, \omega)\left|\Psi_{\nu}(\mathbf{r})\right|^{2} d \mathbf{r}+\frac{c}{2 L} \ln \left(\frac{1}{r_{0}(\omega) r_{1}(\omega)}\right),
\end{aligned}
$$

with $g^{l o c}$ the local material gain and $\alpha$ the free carrier absorption loss.

Edge-emitting lasers such as Fabry-Perot, DFB and DBR lasers have an extreme aspect ratio with a device length much longer than the cross-section diameter. Consequently, the electromagnetic waves propagate mainly along the longitudinal direction and it is a good approximation to consider TE and TM waves only. The optical modes are solutions of the reduced vectorial wave equation ${ }^{13}$ for the transverse field ${ }^{*}$

$$
\left(\nabla^{2}+\frac{\omega_{\nu}^{2}}{c^{2}} \varepsilon\left(\boldsymbol{r}, \omega_{\nu}\right)\right) \Psi_{\nu}(\mathbf{r})=0
$$

where the parametric time-dependence of $\Psi_{\nu}$ has been dropped for notational convenience. Since the direct solution of Eqn. (15) in three dimensions is too complex for the above mentioned laser types, we will motivate a solution strategy based on a separation ansatz.

A general multi-section laser diode consists of several active and passive sections which may have an arbitrary grating corrugation. Additionally, a section with a modestly tapered waveguide is also possible. As an example we mention a SGDBR laser monolithically integrated with a (tapered) semiconductor optical amplifier (SOA) ${ }^{\dagger}$ as depicted in Fig. 1. It can be assumed that during operation the relevant laser parameters vary continuously within each section whereas discrete changes across section interfaces are possible, e.g. at active-passive transitions. In this case we can make the following separation ansatz

$$
\Psi_{\nu}(\boldsymbol{r})=\Phi_{\nu_{\omega}}(x, y ; z) E_{\omega}(z) .
$$

To account for the longitudinal dependence of the dielectric constant $\epsilon(\boldsymbol{r}, \omega)$ due to the inhomogeneous current and temperature distribution, each section is sampled at several positions along the $z$-axis. In this approach we assume a hypothetical waveguide which coincides locally with the actual waveguide. The thus called local normal modes are not themselves solutions of Maxwell's equations since their parameters are functions of $z$. However, they can be superimposed to yield a solution of Maxwell's equations that represents the field of the actual waveguide. ${ }^{15}$ The local character of the corresponding $\nu$-th order transverse mode pattern at oscillation frequency

\footnotetext{
${ }^{*}$ In the derivation of Eqns. (10) and (15) the $\nabla(\nabla \cdot \boldsymbol{E})$ term, which arises from the vector identity $\nabla \wedge \nabla \wedge \boldsymbol{E}=$ $\nabla(\nabla \cdot \boldsymbol{E})-\nabla^{2} \boldsymbol{E}$ has been neglected. ${ }^{14}$

${ }^{\dagger}$ Similar Devices have been fabricated at the University of Santa Barbara.
} 


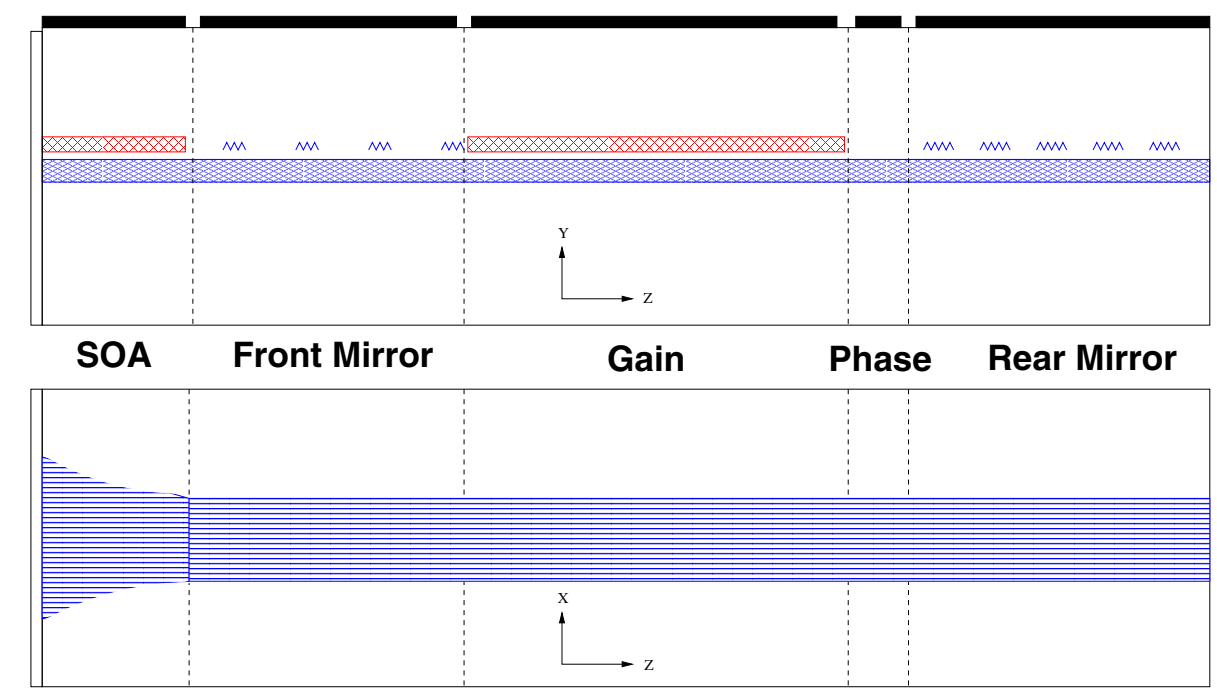

Figure 1. Schematic representation of the longitudinal side view (top) and top view (bottom) of a SGDBR-SOA laser. The top view indicates a tapered waveguide in the SOA section.

$\omega$ is indicated by the colon-separated $z$-dependence of $\Phi_{\nu_{\omega}}$. $E_{\omega}(z)$ stands for the longitudinal electric field. The reduced vectorial wave equation (15) then decomposes into a finite number of two-dimensional Helmholtz equations

$$
\left(\nabla^{2}+\frac{\omega^{2}}{c^{2}}\left[n^{2}(\mathbf{r}, \omega)-n_{\nu_{\omega}}^{2}(z)\right]\right) \Phi_{\nu_{\omega}}(x, y ; z)=0
$$

and a one-dimensional longitudinal cavity problem of the form

$$
\left(\nabla^{2}+\beta^{2}(z)\right) E_{\omega}(z)=0
$$

where the dielectric constant in Eqn. (17) has been expressed in terms of the complex refractive index according to the relation $\varepsilon(\boldsymbol{r}, \omega)=n^{2}(\boldsymbol{r}, \omega)$. The optical material properties are described by the complex refractive index $n(\boldsymbol{r}, \omega)=n^{\prime}+i n^{\prime \prime}$ which relates the optical to the electronic equations. Its imaginary part $n^{\prime \prime}$ contains both local material gain / loss $g^{l o c}(\boldsymbol{r}, \omega)$ in the active region due to stimulated emission / direct inter-band absorption and different kinds of absorption losses $\alpha(\boldsymbol{r}, \omega)$ in non active regions. Then the imaginary part of the index of refraction can be written as

$$
n^{\prime \prime}=\frac{c}{\omega}\left(g^{l o c}-\alpha\right),
$$

with $g^{l o c}$ and $\alpha$ generally depending on carrier density, temperature and frequency. The same dependencies also hold for the real part $n^{\prime}$ when the Kramers-Kroenig relations ${ }^{16}$ are taken into account. In our simulations we model the dominant spectral dependence of the carrier-induced complex refractive index change by ${ }^{17-19}$

$$
\begin{aligned}
\Delta n^{\prime} & =-\frac{e^{2}}{2 n^{\prime} \epsilon_{0} \omega^{2}}\left(\frac{n}{m_{e}}+\frac{p}{m_{h}}\right) \\
\Delta n^{\prime \prime} & =-\frac{e^{3}}{2 n^{\prime} \epsilon_{0} \omega^{3}}\left(\frac{n}{m_{e}^{2} \mu_{e}}+\frac{p}{m_{h}^{2} \mu_{h}}\right) .
\end{aligned}
$$

As an alternative, a lookup-table approach compiled from microscopic calculations is also possible.

In the above Helmholtz equation (17) the propagation constant $\gamma_{\nu_{\omega}}=\frac{\omega}{c} n_{\nu_{\omega}}$ can be identified as the eigenvalue and $n_{\nu_{\omega}}$ as the effective mode index of the local waveguide whose real part is the effective refractive index and 

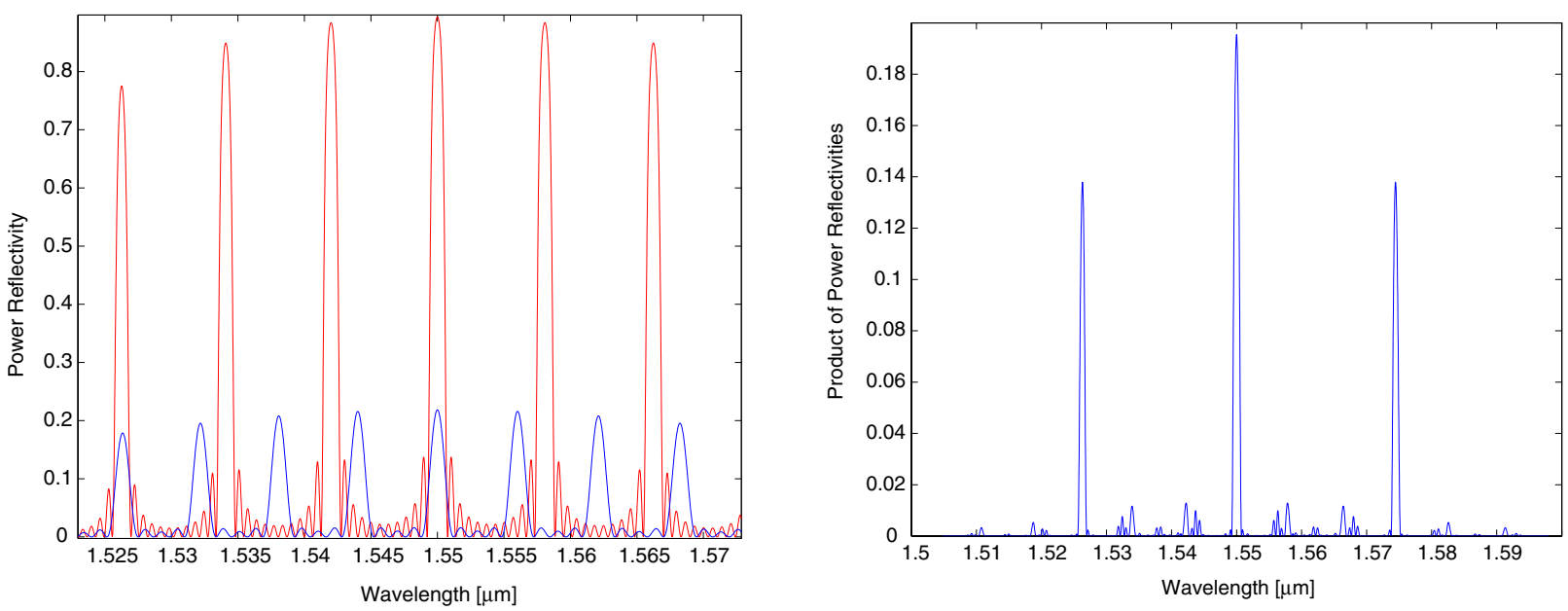

Figure 2. Left: Reflectivity spectrum of the front (blue) and rear (red) sampled-grating mirrors exhibiting the characteristic periodic reflection peaks in the desired wavelength region. Right: Product of front and rear mirror reflectivities indicating the main mode together with the dominant side-modes when the mirrors are perfectly aligned with respect to their center-peaks.

the imaginary part is related to the net modal gain by $g_{n e t}=\frac{\omega}{c} \operatorname{Im}\left(n_{\nu_{\omega}}\right)$. In the longitudinal cavity problem $\beta$ reads as

$$
\beta(z)=\frac{\omega}{c} n_{\nu_{\omega}}(z)+i \frac{\Delta \alpha}{2},
$$

where $\Delta \alpha$ is the net power-loss of the cavity.

Eqns. (17) and (18) together with the appropriate boundary conditions, standard Dirichlet / Neumann boundary conditions and Sommerfeld radiation boundary condition, ${ }^{20}$ respectively, determine the pairs $\left(\Psi_{\nu}, \omega_{\nu}\right)$ of resonant modes and frequencies of the laser cavity. In order to suppress a net contribution to the longitudinal and temporal dependence of $\Psi_{\nu}(\boldsymbol{r} ; t)$, the local transverse mode pattern $\Phi_{\nu_{\omega}}$ and the longitudinal field distribution $E_{\omega}$ have to be normalized according to ${ }^{7}$

$$
\begin{aligned}
\iint\left|\Phi_{\nu_{\omega}}(x, y ; z)\right|^{2} d x d y & =1, \quad \forall z \epsilon[0, L], \\
\int_{0}^{L}\left|E_{\omega}(z)\right|^{2} d z & =1
\end{aligned}
$$

where $L$ is the cavity length.

It should be noted that a more rigorous approach would calculate/cover the coupling between all local normal modes of neighboring longitudinal positions. In our approach we make two approximations. First, we consider only coupling between neighboring normal modes of the same order. Secondly, the one-dimensional wave equation (18) essentially describes power coupling of the modes along the $z$-direction. These assumptions greatly simplify the analysis while little accuracy is lost for the aforementioned laser structures.

We believe that the approach outlined in this section is well suited for the efficient simulation of general active structures such as sampled-grating DBR lasers, ${ }^{21}$ tapered waveguide lasers ${ }^{22,23}$ and other monolithically integrated optoelectronic devices.

\section{NUMERICAL IMPLEMENTATION}

In this section we will highlight on the numerical implementation of the optical equations outlined in Sect. 2.2 with respect to modeling the wavelength tuning behavior of multi-section DBR lasers. In contrast to standard 
Fabry-Perot laser diodes the emission wavelength can no longer be assumed to coincide with the modal gain peak. Depending on the amount of current injected into the grating sections of a SGDBR laser, the lasing wavelength can be tuned over a range of up to $100 \mathrm{~nm}$. What's more, discrete mode/wavelength jumps are possible and the convergence criteria have to be reviewed. Since the product of the power reflectivities of the front and rear mirror at the resonant wavelength varies during tuning operation, the corresponding mirror loss and threshold gain are subject to change. At last, the influence of the tuning operation on the optical mode pattern should also be mentioned here.

The strong coupling of the optics and electronics, characterized by the several-orders-of-magnitude change in optical intensity across threshold, is treated by incorporating the photon rate equation (10) into a Newton updating scheme. ${ }^{7}$ What is usually referred to as weak coupling in Fabry-Perot laser simulation, i.e. the dependence of the electro-thermal equations together with the photon rate equations on the optical field distribution and the lasing wavelength, does not hold strictly true for SGDBR lasers. However, experience shows that a modified Gummel-type iteration scheme between Eqns. (1)-(4) and Eqn. (15) can be employed to obtain a fully self-consistent solution of the whole nonlinear system. The necessity for a modified Gummel-type iteration scheme will be explained later in this section.

Solving Eqn. (15) by means of the separation ansatz (16) requires the solution of two different classes of eigenvalue problems. The Helmholtz equation (17) is a standard non-Hermitian eigenvalue problem of the form $A x=\lambda x$, with $\lambda \in \mathbb{C}$. For the discretization of the Helmholtz equation a finite-element method is employed as

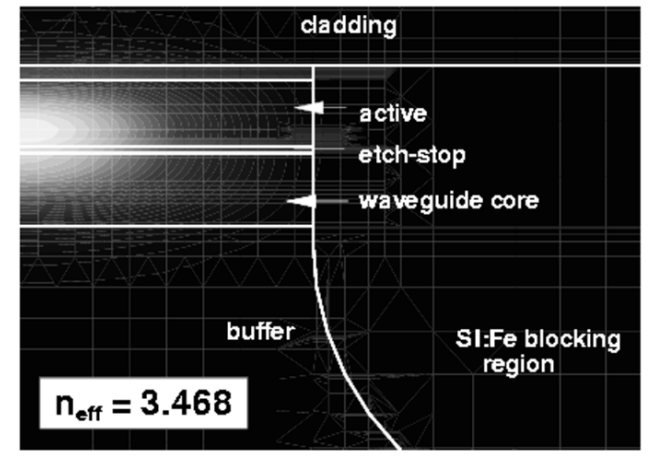

a)

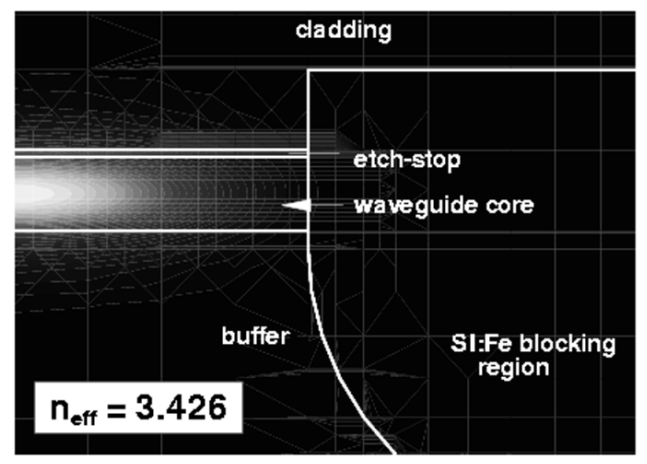

c)

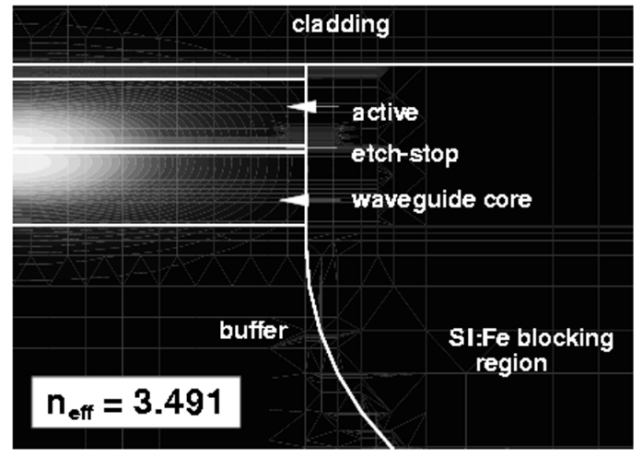

b)

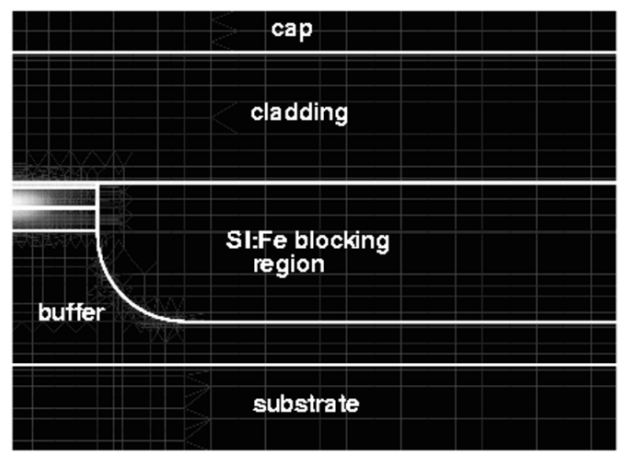

d)

Figure 3. Optical field patterns of the fundamental transverse mode at various positions along the waveguide: (a) gain section, (b) transition region between gain and phase section, (c) passive section. For better illustration, graphs (a)-(c) only show a display detail of $(\mathrm{d})$ where the optical confinement factor is high. 
presented in detail in Ref. 24. On the other hand, the longitudinal equation (18) represents a generalized nonhermitian nonlinear eigenvalue problem of the form $A x=\xi B(\xi) x$ with the complex eigenvalue $\xi=\omega / c+i \Delta \alpha$. Although we mostly deal with periodic grating corrugations which can be treated analytically in the framework of coupled mode theory, ${ }^{25-27}$ the inhomogeneous carrier and temperature distribution during laser operation demands for a numerical solution. To this end a transfer-matrix formalism has been chosen that relates the right- and left-propagating waves at one point of the structure to the right- and left-propagating waves at another point ${ }^{28,29}$

$$
\left(\begin{array}{c}
E^{r}\left(z_{i}\right) \\
E^{l}\left(z_{i}\right)
\end{array}\right)=\left(\begin{array}{ll}
T_{11} & T_{12} \\
T_{21} & T_{22}
\end{array}\right)_{i}\left(\begin{array}{c}
E^{r}\left(z_{i+1}\right) \\
E^{l}\left(z_{i+1}\right)
\end{array}\right)
$$

Cascading all transfer matrices yields a system matrix $\boldsymbol{T}=\prod^{i} T_{i}$. Applying the Sommerfeld radiation condition ${ }^{20}$ requires $\boldsymbol{T}_{22}=0$ and hence the problem reduces to a complex root search for which a variant of the Nonlinear Simplex (Nelder-Mead) Algorithm ${ }^{30}$ has been employed.

For the solution of the one-dimensional cavity equation the effective mode index distribution is superimposed with the grating corrugation $\Delta n_{g r}(z)$. In this way, Eqn. (18) can be solved for an arbitrary variation in the superimposed effective mode index if the discretization of the $z$-axis is chosen small enough so that $n_{\nu_{\omega}}^{\text {tot }}(z)=$ $n_{\nu_{\omega}}(z)+\Delta n_{g r}(z)$ is well approximated by a piecewise constant function. As the effective mode index $n_{\nu_{\omega}}$ generally is a slowly varying function except at active-passive section interfaces, a coarser sampling of the $z$-axis in combination with a linear interpolation is permissible.

In a general multi-mode simulation we have to consider several longitudinal modes for each higher order local transverse mode. Furthermore, in order to include dispersion Eqn. (17) has to be solved at each sampling position for a range of frequencies within which the longitudinal resonant frequencies with lowest threshold gain are expected. In this context the frequency $\omega$ can be seen as an input parameter in the Helmholtz equation and a position- and frequency-dependent function $n_{\nu_{\omega}}(z)$ can be compiled. The actual frequencies of the lasing modes are then obtained from the longitudinal cavity problem. For single longitudinal-mode simulations dispersion

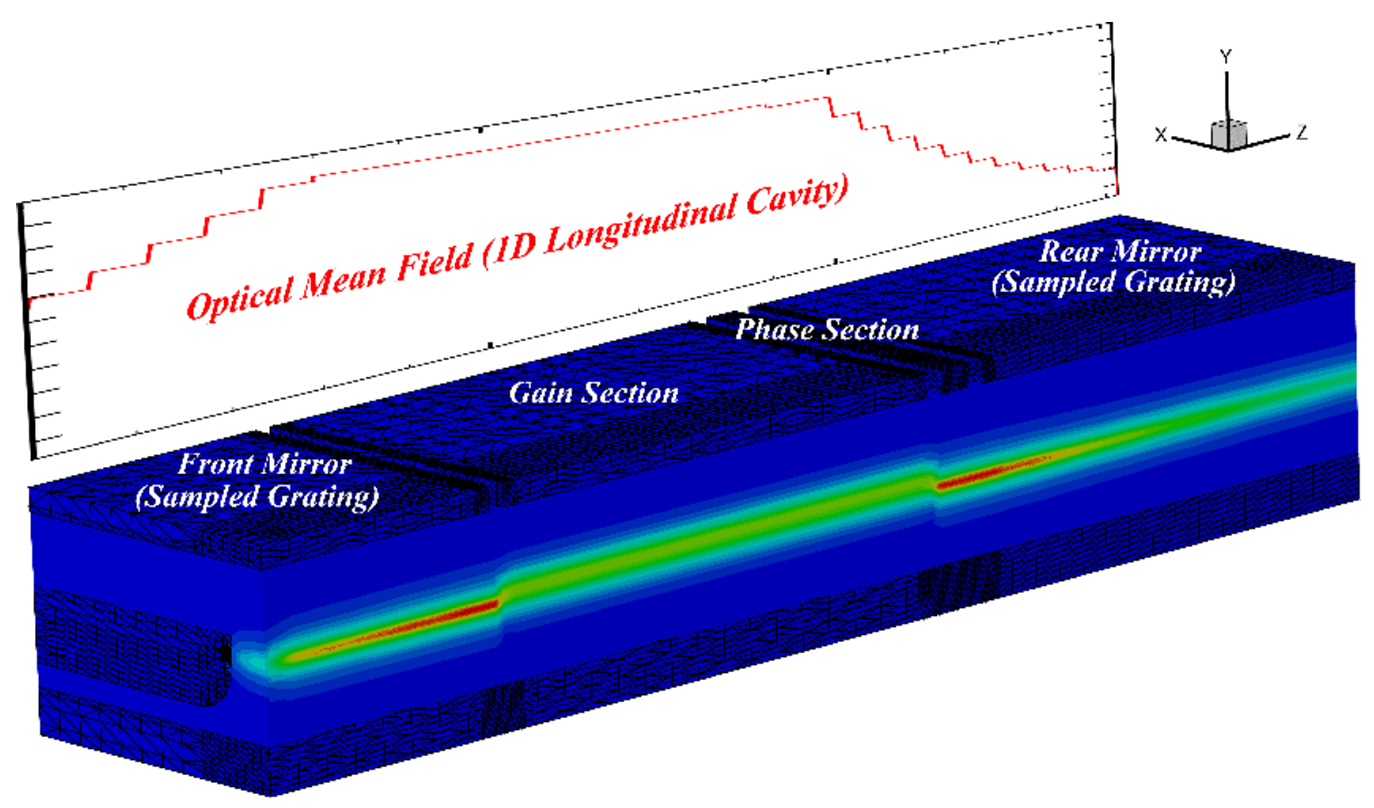

Figure 4. Optical intensity distribution of a planar buried-heterostructure SGDBR laser resulting from a separation ansatz with parametric $z$-dependence of the local transverse modes as described in Sect. 2.2. The longitudinal intensity envelope at transparency is given in the backdrop. The buried-heterostructure type SGDBR laser has only been used for illustration of the 3D optical model. 


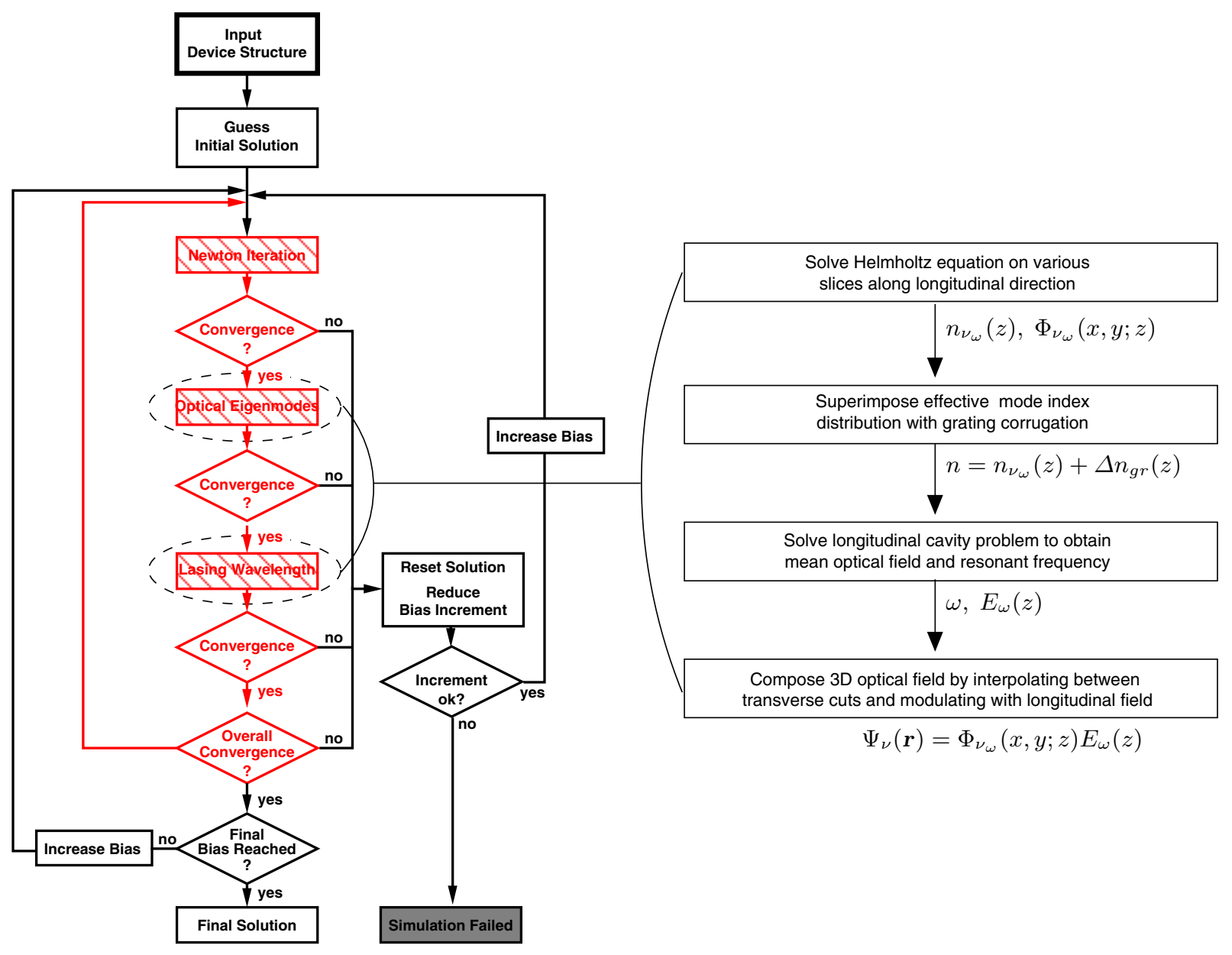

Figure 5. Simulation flow for self-consistent simulation of tunable multi-section DBR lasers in three dimensions. The right part of the chart highlights the necessary steps for the computation of the optical eigenmodes and the lasing frequency.

can also be included using a Taylor expansion of the complex refractive index at a target frequency $\omega_{0}$ in the neighborhood of the resonant frequency

$$
n\left(\boldsymbol{r}, \omega_{0}\right)=n\left(\boldsymbol{r}, \omega_{0}\right)+\left.\omega_{0} \frac{\partial n}{\partial \omega}\right|_{\omega_{0}} .
$$

In a multi longitudinal-mode setting this approximation may no longer be valid if the difference between the target frequency $\omega_{0}$ and the side-mode frequency $\omega_{1}$ is big as is typically the case in SGDBR lasers.

In the derivation of the photon rate equation and the corresponding optical eigenvalue problems the imaginary part $\Delta \alpha$ of the eigenvalue in Eqn. (18) can be identified ${ }^{31}$ with the difference term $\left(G_{\nu}-L_{\nu}\right)$ in the photon rate equation. However, on numerical grounds it is advantageous to calculate this term using the integral approximations (12) and (14) due to the non-local nature of the eigenvalue. This allows for the calculation of the local partial derivatives in the Jacobian matrix which guarantees the convergence properties of the Newton method.

The simulation flow for a general three-dimensional simulation of a tunable multi-section DBR laser is illustrated in Fig. 5. At each bias point the coupled system of PDEs and the optical eigenmodes along with the lasing wavelength are solved self-consistently in a Gummel-type iteration scheme. ${ }^{32}$ The solution of the system of PDEs using a Newton-Raphson scheme is followed by the computation of the optical eigenmodes and lasing wavelength as summarized on the right in Fig. 5. Due to the interaction between optics and electronics this 
procedure has to be repeated at least once in order to ensure overall convergence. The convergence criteria are based on the solution errors with respect to the last Newton iteration in the case of the coupled PDEs and to the respective solution of the previous Gummel-type iteration step for the other parts.

While the solution scheme described above works well for many laser structures, tunable multi-section DBRtype lasers sometimes require a modified solution strategy. They are different in that the lasing wavelength is determined not only by the effective cavity mode with the highest gain, as in Fabry-Perot lasers, but also by the overlap with the continuous grating reflectivity spectrum. In this mechanism the waveguide phase plays a crucial role. Therefore DBR lasers usually contain a phase shifter section which allows for the independent control of the cavity mode locations and hence a quasi-continuous tuning behavior. In certain simulation configurations of SGDBR lasers the sensitivity of the waveguide phase with respect to the carrier density and temperature distributions can cause an oscillation between either adjacent cavity modes or adjacent channel modes ${ }^{\ddagger}$ in consecutive iterations of the Gummel loop. This effect is due to numerical errors, e.g. interpolation errors, and can be alleviated with a damping of the optical modes in the iteration scheme once the relative error has decreased to the point where the oscillatory behavior begins. Earlier on we referred to this as a modified Gummel-type iteration.

In order to exploit the optimal wavelength tuning behavior careful adjustment of the phase section current is necessary. In real-world applications this can be done either interactively or by an electronic control loop calibrated through an extensive device characterization process. For obvious reasons neither of them is an option for a device simulator. The approach chosen in this work involves the following tasks in each bias step:

- calculate the front and rear mirror reflectivity spectra $r_{0}(\omega)$ and $r_{1}(\omega)$,

- determine the frequency $\omega_{0}$ for which the product of the mirror reflectivities $R(\omega)=r_{0}(\omega) \cdot r_{1}(\omega)$ has a global maximum,

- take the frequency $\omega_{0}$ as an input to Eqn. (18) and perform a two-dimensional root search with respect to $\left(\Delta n_{p h}^{\prime}, \Delta \alpha\right)$, where $\Delta n_{p h}^{\prime}$ denotes the real part of the refractive index change in the phase section ,

- keep $\Delta n_{p h}^{\prime}$ fixed and calculate the remaining longitudinal side modes.

This ensures the alignment of the comb-mode spectrum with the reflectivity peak. The calculated required index change in the phase section can then be used to extract the corresponding change in the injection current.

\section{RESULTS}

In this work we have analyzed an InP based ridge-waveguide SGDBR laser with an active region consisting of seven compressively strained InGaAsP quantum wells ${ }^{\S}$. Further details on the device structure can be found in the table in Fig. 7. A hierarchical approach ${ }^{33}$ has been followed for the efficient calibration of this complex laser device. First, the material parameters of the active section were calibrated by way of $1 \mathrm{D}$ and $2 \mathrm{D}$ simulations. To investigate the tuning behavior such as the dependence of the lasing wavelength, output power, loss and active section voltage on the mirror currents, 3D simulations are necessary.

In an initial simulation step we ramp to the operation point, as shown in Fig. 6, whose state can be saved and reloaded as an initial solution in other simulations. Then we increment for example the front mirror current to a fixed value and sweep the rear mirror current. In this way we generated the tuning map shown in Fig 7 on the right. It was obtained by sweeping the rear mirror current for 40 different values of the front mirror current. This task lends itself to be distributed over a computer cluster where it can be accomplished in only 5 hours . The simulated tuning map is in good agreement with experimental data shown on the left in Fig. 7. Further

\footnotetext{
${ }^{\ddagger}$ For a description of possible competing modes in a SGDBR laser see Ref. 27.

$\S$ The device under investigation has been fabricated at the University of California in Santa Barbara.

${ }^{\top}$ We used a Linux cluster consisting of 20 nodes each featuring a Dalco r2164ia, 2x2.4 GHz AMD Opteron_250/1MB Cache CPU. Each simulation required about $2 \mathrm{~GB}$ RAM.
} 


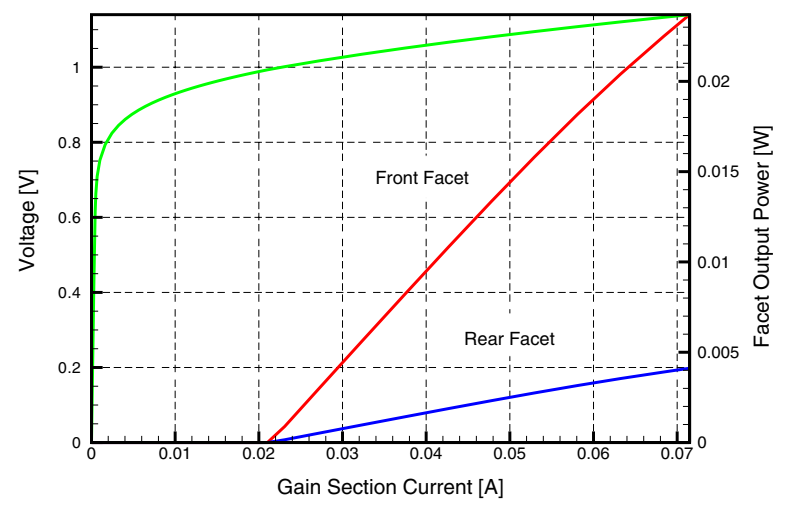

Figure 6. Optical output power and gain section voltage versus terminal current characteristics as obtained from full three-dimensional simulation.
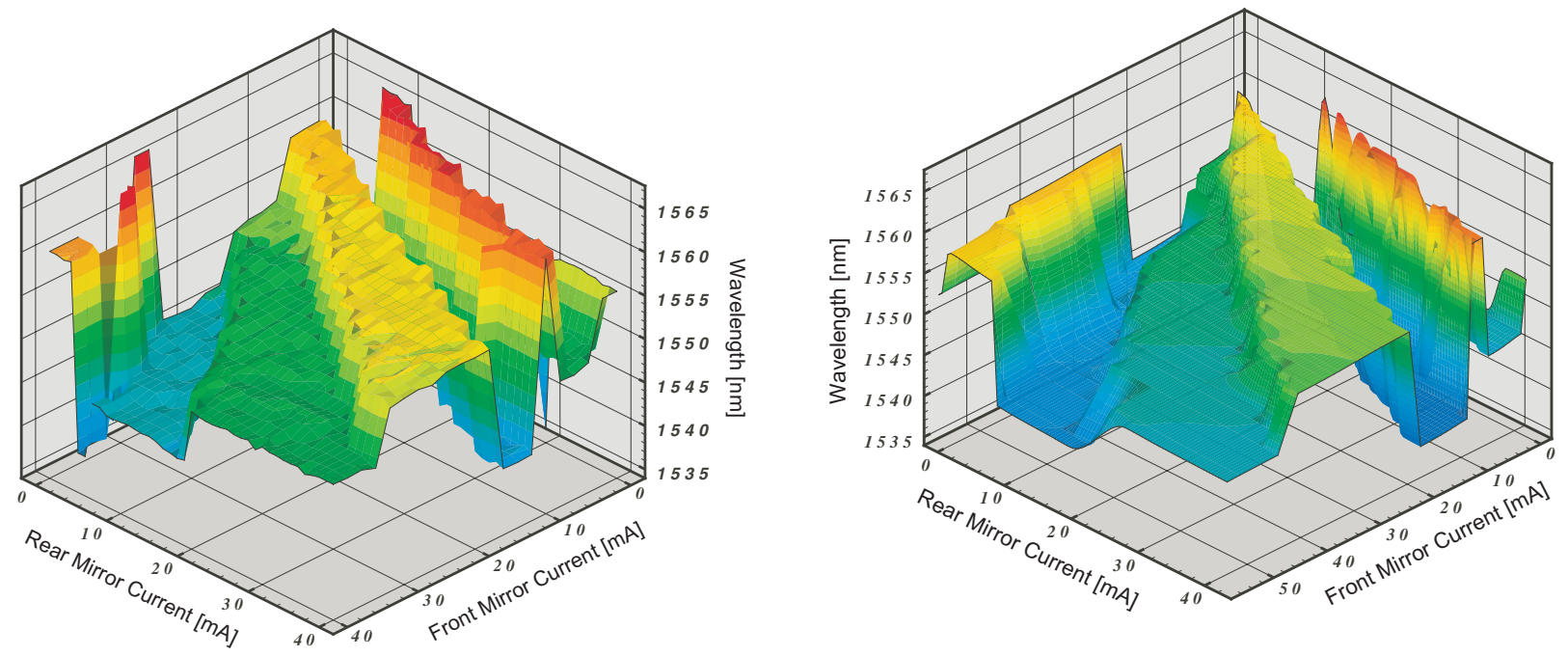

Device Parameters

\begin{tabular}{|l|l|c|c|}
\hline Section Name & $\mathrm{L}[\mu \mathrm{m}]$ & $\kappa[1 / \mathrm{cm}]$ & Sampled Grating DBR \\
\hline \hline Front Mirror & 307.5 & 250 & $5 \times 4 \mu \mathrm{m}$ grating bursts with $46 \mu \mathrm{m}$ period \\
\hline Gain & 500 & - & - \\
\hline Phase & 75 & - & - \\
\hline Rear Mirror & 552 & 250 & $12 \times 6 \mu \mathrm{m}$ grating burst with $65.5 \mu \mathrm{m}$ period \\
\hline
\end{tabular}

Figure 7. Wavelength tuning behavior of SGDBR laser as specified in the table (bottom). Comparison of tuning maps obtained from measurement (left) and from 3D simulation (right) shows very good agreement.

improvement can be achieved by a better calibration of the carrier dependence of the refractive index model. This essentially determines the scaling of the tuning map.

In addition to the wavelength tuning map the corresponding maps for the optical output power, loss and active section voltage can be analyzed. These properties are of importance to developing mode control algorithms, i.e. finding the optimal section currents for each wavelength channel. 


\section{CONCLUSIONS}

Following a hierarchical approach to the general multidimensional laser simulation, the tuning behavior of a SGDBR laser has been investigated and close agreement with measurements has been achieved. It has been shown that choosing a suitable solution method for the optical problem allows for full three-dimensional simulations for such complex device structures without any approximations with regards to longitudinal carrier transport. Furthermore, with a modern computer infrastructure at hand a comprehensive analysis can be performed within the order of a few hours. This opens the door for design and optimization tasks even in the scope of a device engineer.

\section{ACKNOWLEDGMENTS}

We would like to thank John Hutchinson from Intel Corporation for performing the measurements of the SGDBR laser. This work has been funded by the Swiss Technology Oriented Program NANO21, Project No. 5785.1.

\section{REFERENCES}

1. M. L. Masanovic, V. Lal, J. A. Summers, J. S. Barton, E. J. Skogen, L. A. Coldren, and D. J. Blumenthal, "Design and performance of a monolithically integrated widely tunable all-optical wavelength converter with independent phase control," IEEE Photonics Technology Letters 16(10), pp. 2299-2301, 2004.

2. M. N. Sysak, J. S. Barton, L. A. Johansson, J. W. Raring, E. J. Skogen, D. J. Masanovic, M. L.and Blumenthal, and L. A. Coldren, "Single-chip wavelength conversion using a photocurrent-driven EAM integrated with a widely tunable sampled-grating DBR laser," IEEE Photonics Technology Letters 16(9), pp. 20932095, 2004.

3. J. Piprek, S. Li, P. Mensz, and J. Hader, "Monolithic Wavelength Converter: Many-Body Effects and Saturation Analysis", Chapter 14 in: Optoelectronic Devices - Advanced Simulation and Analysis, Springer Verlag, New York, 2005.

4. D. L. Scharfetter and H. K. Gummel, "Large-signal analysis of a silicon Read diode oscillator," IEEE Trans. Elec. Dev. 16, pp. 64-77, 1969.

5. Y. Apanovich, E. Lyumkis, B. Polsky, A. Shur, and P. Blakey, "Steady-state and transient analysis of submicron devices using energy balance and simplified hydrodynamic models," IEEE Transactions on ComputerAided Design of Integrated Circuits and Systems 13, pp. 702-711, June 1994.

6. Y. Apanovich, P. Blakey, R. Cottle, E. Lyumkis, B. Polsky, A. Shur, and A. Tcherniaev, "Numerical simulation of submicrometer devices including coupled nonlocal transport and nonisothermal effects," IEEE Transactions on Electron Devices 42(5), pp. 890-898, 1995.

7. B. Witzigmann, A. Witzig, and W. Fichtner, "A multidimensional laser simulator for edge-emitters including quantum carrier capture," IEEE Trans. ED 47, pp. 1926-1934, 2000.

8. B. Witzigmann, A. Witzig, and A. Fichtner, "A full 3-dimensional quantum-well laser simulation," in Proceedings of LEOS, 2000.

9. M. Pfeiffer, A. Witzig, and W. Fichtner, "Coupled electro-thermo-optical 3D simulation of edge-emitting lasers," in Proceedings of the NUSOD, 2001. Full presentation to be found at: http://www.iis.ee.ethz.ch/ laser/witzig.handout.ps.

10. R. E. Bank, D. J. Rose, and W. Fichtner, "Numerical methods for semiconductor device simulation," IEEE Trans. Elec. Dev. 30(9), pp. 1031-1041, 1983.

11. G. Baraff and R. Smith, "Nonadiabatic semiconductor laser rate equations for the large-signal, rapidmodulation response regime," Phys. Rev. A 61, pp. 1-13, 2000.

12. X. Li, A. D. Sadovnikov, W.-P. Huang, and T. Makino, "A physics-based three-dimensional model for distributed feedback laser diodes," IEEE Journal of Quantum Electronics 34, pp. 1545-1553, September 1998.

13. R. Courant and D. Hilbert, Methods of Mathematical Physics, vol. 2, Interscience Publishers, New York, 1962.

14. D. Marcuse, Light Transmission Optics, Robert E. Krieger Publishing Company, second edition ed., 1989.

15. D. Marcuse, Theory of Dielectric Optical Waveguides, Academic Press, 1974.

16. J. D. Jackson, Classical Electrodynamics, John Wiley \& Sons, Inc., New York, second. ed., 1975. 
17. C. H. Henry, R. A. Logan, and K. A. Bertness, "Spectral dependence of the change in refractive index due to carrier injection in GaAs lasers," Journal of Applied Physics 52, pp. 4457-4461, 1981.

18. B. R. Bennett, R. A. Soref, and J. A. D. Alamo, "Carrier-induced change in refractive index of InP, GaAs, and InGaAsP," IEEE J. Quantum Electron. 26, pp. 113-122, 1990.

19. J.-P. Weber, "Optimization of the carrier-induced effective index change in InGaAsP waveguides - application to tunable bragg filters," IEEE J. Quantum Electron. 30(8), pp. 1801-1816, 1994.

20. A. Sommerfeld, Partial Differential Equations in Physics, Academic Press, 1949.

21. B. Mason, G. A. Fish, S. Denbaars, and L. Coldren, "Widely tunable sampled grating DBR laser with integrated electro-absorption modulator," IEEE Photonics Technology Letters 11(6), pp. 638-640, 1999.

22. T. L. Koch, U. Koren, G. Eisenstein, M. G. Young, M. Oron, C. R. Giles, and B. I. Miller, "Tapered waveguide InGaAs/InGaAsP multiple-quantum-well lasers," IEEE Photonics Technology Letters 2(2), pp. 88-90, 1990.

23. M. Chien, U. Koren, B. I. Miller, M. Oron, M. G. Young, and J. L. Demiguel, "Short-cavity distributed Bragg reflector laser with an integrated tapered output waveguide," IEEE Photonics Technology Letters 3(5), pp. 418-420, 1991.

24. J. Jin, The Finite-Element Method in Electromagnetics, Wiley, 1993.

25. A. Yariv and P. Yeh, Optical Waves in Crystals, John Wiley \& Sons Inc., 1984.

26. G. P. Agrawal and N. K. Dutta, Long-Wavelength Semiconductor Lasers, Van Nostrand Reinhold, 1986.

27. V. Jayaraman, Z.-M. Chuang, and L. A. Coldren, "Theory, design and performance of extended tuning range semiconductor laser with sampled gratings," IEEE J. Quantum Electron. 29(6), pp. 1824-1834, 1993.

28. H. Kogelnik and C. V. Shank, "Coupled-wave thoery of distributed feedback lasers," J. Appl. Phys. 43(5), pp. 2327-2335, 1972.

29. M. Yamada and K. Sakuda, "Analysis of almost-periodic distributed feedback lasers," Applied Optics 26, pp. 3474-3478, 1987.

30. J. A. Nelder and R. Mead, "A simplex method for function minimization," Computer Journal 7, pp. 308-313, 1965.

31. C. H. Henry and R. F. Kazarinov, "Quantum noise in photonics," Reviews of Modern Physics 68, pp. 801853, July 1996.

32. H. K. Gummel, "A self-consistent iterative scheme for 1-dimensional steady state transistor calculations," IEEE Trans. Elec. Dev. 11(10), p. 455, 1964.

33. L. Schneider, M. Pfeiffer, M. Streiff, A. Witzig, and W. Fichtner, "Full-3D simulation of tunable multisection DBR lasers," in Proceedings of the NUSOD, 2003. Full presentation to be found at: http://hotaka.t.u-tokyo.ac.jp/nusod03/images/presentation/ThC3.pdf. 